\title{
INVERSED MATRIXES IN ELECTRIC CIRCUITS PARAMETERS ESTIMATION BY ORIENTED GRAPHS
}

The base of electric circuit analysis is often set up on numerical methods. When taking a look into the principle of any numerical method, a matrix calculus always rises out. The larger the problem is, the larger is the size of the matrix, representing the logical structure of the solved problem [1]. The problem (especially from the mathematical point of view) is in acquiring the inverse matrix, which can be solved with some of a large scale of available numerical (or analytical; based on the size of matrixes) methods. This paper shows another possible approach to obtaining the inverse matrix. The graph theory has already been successfully used to invert the matrixes; although, the idea of evaluating the alternating paths, presented by authors, is completely new and had not been used before. The utilization of the designed procedure is demonstrated on a part of an analogue signal processing filter. This technology gives a new tool to software designers and could speed up the numerical solutions; or, in case of impossible analytical and/or diverge numerical technique can lead to a successful and reasonable solution.

Keywords: Invert of matrixes, mathematical representation of electric circuits, graph theory.

\section{Introduction}

Graphs are historically and generally considered as geometrical objects. In spite of this, they can be represented in many different ways: lists of edges, adjacency matrixes or by a lot of other different structures [2]. The reality represented by graphs can be anything between chemical formulae, mechanical system, map structure and/or computer processes. Graphs are visually impressive and can easily introduce the observer into the core of the problem.

The paper will introduce the necessary base of graph theory to the readers and describe the inverse matrix acquisition based on graph theory. This means that neither numerical nor analytical method is used [3, 4]. This new approach can both spare computational time (for certain types of problems) and lead to solution where numerical or analytical methods cannot be used. In addition, the presented technology can be implemented into the cores of numerical (or analytical) tools to obtain the required solution of the matrix formulated problem. The paper is based on latest research of authors presented in [5] and completed with description of background theory and presenting new and completed examples.

The paper describes the principles of topological description of electric circuits based on graphs theory. The electric circuit, described by its topology, could easily be represented by graphs. The equivalent circuit of an asynchronous rotary machine can be presented as an important and well-known example (Fig. 1). The parameters from Fig. 1 are generally known and it is not necessary to describe them in this paper. Important is that the edges in Fig. 1 are easy to identify. The result of this assignment can be seen in Fig. 2. These steps might be successfully used for almost every rotary or linear [6] machinery properties analysis.

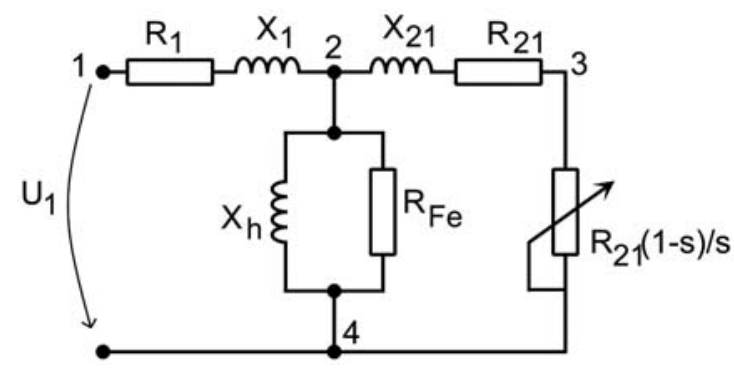

Fig. 1 Equivalent circuit of an asynchronous machine

The utilization of different graph types in electric circuits' analysis is not a new idea at all. There are technologies (based either on oriented graphs or directly describing the topological structure of the circuit) used parallel to numerical or analytical techniques [7]. One of the most referred are signal graphs [5]. Signal Graphs are mapping the flow of electrical signals through the given circuit. They are able to interpret the graphical representation of Kirchhoff's Laws; nevertheless, they are not able to follow the topology of the circuit. The signal graph, following the given rules, can be simplified together with a change of individual legs

\footnotetext{
* Sona Pavlikova1, Dusan Maga2, Boris Simak2

${ }^{1}$ Slovak University of Technology, Bratislava, Slovakia, E-mail: sona.pavlikova@stuba.sk

${ }^{2}$ Czech Technical University in Prague, Prague, Czech Republic, dusan.maga@fel.cvut.cz
} 
transformation. Finally, the algebraic expressions are obtained from the graph structure. The investigated parameters can easily be evaluated according to a numerical (or analytical - where possible) solution of equation sets representing Kirchhoff's laws.

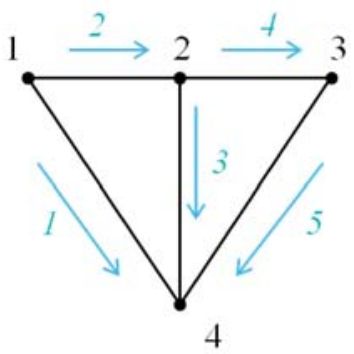

Fig. 2 Edges of the circuit

\section{Topological graphs}

In contrast to oriented signal graphs, this type of graphical structure represents directly the topological structure of the circuit. The vertices are the nodes and the edges are the arms (Fig. 1). The topological graph can be used for analytical representation of a circuit's topological structure in the form of matrix. The matrixes obtained from these are known as Incidence topological matrixes.

To be able to build the topological graph of the circuit, it is necessary to replace the dipole components of the circuit with their representative - edge of the graph. The most important term in graph theory is the graph tree. This definition covers such a subgraph, which contains all of the vertices, but no closed cycle. The edges of the graph forming the tree are called arms, while the complementary edges are called chords.

The graphical connection can be transformed into an algebraic representation with several types of topological matrixes.

\section{Incidence matrix}

The adjacency matrix of oriented graph with vertices $v_{1}, \ldots, v_{n}$ is a square matrix $A(G)=\left(a_{i j}\right)$ of n-th degree, where $a_{i j}$ is equal to number of edges between vertices $v_{i}$ and $v_{j}$. The incidence matrix $M=\left(m_{i j}\right)$ is a matrix with size of $p \times q$, where $\mathrm{p}$ represents the number of vertices and $\mathrm{j}$ number of edges. The value of $m_{i j}$ is equal either to 1 or 0 - based on incidence of vertex $i$ and edge $j$. It is also important to define the diagonal matrix $D m_{i j}\left(d_{i j}\right)$, where value of $d_{i j}$ is equal to degree of vertex $i$. The relation between the adjacency matrix and incidence matrix is as presented:

$$
A=D-M M^{T}
$$

The concrete construction of the incidence matrix can be illustrated at an example presented in Fig. 1 and its graph representa- tion (Fig. 2). As can be seen in Fig. 2, there is either one or none connection (edge) between the vertices. The rows of the matrix are based on vertices and the columns on edges of the graph. As described above, each row $i$ of this matrix express which edges are connected to vertex $j$. For example, edge 1 leaves from vertex 1 so $m_{11}$ is equal to -1 . The same goes for edge 2 and vertex $1: m_{12}$ is equal to -1 again. Edges 3, 4 and 5 have no connection (incidence) to vertex No. 1 , so their values are equal to 0 . The edge 2 arrives to vertex 2: the value of $m_{22}$ is equal to 1 . Based on these steps the following matrix is obtained:

$$
\left(\begin{array}{ccccc}
-1 & -1 & 0 & 0 & 0 \\
0 & 1 & -1 & -1 & 0 \\
0 & 0 & 0 & 1 & -1 \\
1 & 0 & 1 & 0 & 1
\end{array}\right)
$$

It is generally known that any row in the incidence matrix is a linear combination of the rest of the rows. This means that one of the rows contains redundant information and it is possible to leave it out of the matrix. It is typical to quit the row corresponding to reference (grounded) node, e.g. the fourth row from the above presented incidence matrix. The obtained type of matrix is called node matrix and is labeled as R. For the case presented in Fig. 2 it is equal to:

$$
R=\left(\begin{array}{ccccc}
1 & -1 & 0 & 0 & 0 \\
0 & 1 & -1 & -1 & 0 \\
0 & 0 & 0 & 1 & -1
\end{array}\right)
$$

When assigning every $n^{\text {th }}$ graph vertex to potential $\varphi_{n}$ (relative to reference node), each single component $a_{n k}$ of matrix $R$ can be considered as an expression of potential $\varphi_{n}$ influence on current flowing through edge $k$. When the voltages on the edges are represented in the form of column vector $u$ and the potentials of vertices in the form of column vector $\varphi$, then the voltage of edges can be calculated as:

$$
u=R^{T} \varphi
$$

The matrix $R$ and the column vector $\underline{u}$ can be separated into parts $R_{v}, R_{t}$ and $u_{v}, u_{t}$. These components correspond to tree arms and chords. The dependence between the potential of each vertex and the voltage of each edge can be obtained from (5).

$$
\left(\begin{array}{l}
R_{v}^{T} \\
R_{t}^{T}
\end{array}\right) \varphi=\left(\begin{array}{l}
u_{v} \\
u_{t}
\end{array}\right)
$$

More detailed description of connection to electric circuits presented by the authors can be seen, e.g. in [5].

\section{Inverse matrix by graph construction}

There are numerous methods used to obtain the inverse matrix. One of them is based on the graph construction. The method is described in a simple example with alternating paths (the term alternative path covers those paths where the edges of 1-factor and edges out of 1-factor are alternating, and it begins and ends with edge out of 1-factor [4]). The matrix $A$ is adjacent to graph $G$. There 
also is an inverse graph $G^{-1}$ with its adjacent matrix $A\left(G^{-1}\right)$. The matrix obtained this way is an inverse matrix to the original one. Graph $G$ is understood as a finite non-oriented graph without loops with the possibility of multiple edges [8]. The labeling of graph $G$ is represented as $l: E(G) \rightarrow R$, where $R$ is the set of real numbers. When labeling $l$ on graph $G$ is available, the definition of adjacent matrix $A=\left(a_{i j}\right)$ to graph $G$ is a square matrix of order $n$, where:

$$
a_{i j}=\left\langle\begin{array}{ll}
l\left(v_{i}, v_{j}\right) & \text { if }\left(v_{i}, v_{j}\right) \in E(G) \\
0 &
\end{array}\right.
$$

Graph $G$ is called bipartite graph (or simply bigraph) when it does not contain a circle of odd length. The non-empty sets $R \subset$ $\subset V(G)$ constitute the bipartition to bigraph $G=(V(G), E(G))$ when $R \cap C=\varnothing$ and $R \cup \mathrm{C}=V(G)$ and none of two vertices from $R$ (or $C$ ) are connected with an edge. When the bipartition $(R, C)$ of bigraph $G$ is given, the $R \times C$ of matrix $B_{R C}(G)=b_{i j}$ can be defined $(|R|$ means cardinality of set $R$ ) as follows:

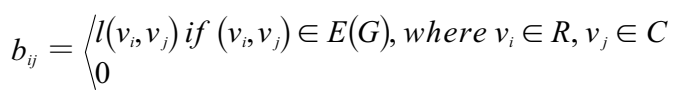

This matrix is called bipartite matrix of graph $G$. It is obvious that:

$$
A(G)=\left(\begin{array}{cc}
0 & B_{R C}^{T}(G) \\
B_{R C}^{T}(G) & 0
\end{array}\right)
$$

Additionally, if $B_{R C}(G)$ is a regular square matrix then also $A(G)$ is regular. Graph $G$ contains 1-factor (perfect matching) if a factor of the graph with each vertex with a degree of 1 exists. Symbol $M(G)$ or $(M)$ will be assigned to 1-factor of graph $G$ (if included in) and symbol $G / M$ to the graph obtained from $G$ by contraction on edges of 1 -factor [9]. The path with $n$ vertices is labeled as $P_{n}$. The path $P$ in $G$ of length $k$ is a subgraph of $G$ isomorphic with $P_{k+1}$. Let $G$ contain 1-factor $M(G)$. The path $P$ is in $G$ alternating in respect of $M(G)$ if one of the following criteria is fulfilled:

- $P$ is of length 1 (i. e. edge),

- from each pair of adjacent edges in $P$ there is just one belonging to $M(G)$ and $P$ begins and ends with an edge not belonging to $M(G)$.

The $P$ is even (odd) alternating path when $P$ contains even (odd) number of edges out of $M(G)$. When $P$ is only an edge, it will be considered even (odd) alternating path if it belongs (does not belong) to $M(G)$.

Let $G$ be a bigraph without multiple edges with unique 1-factor $M(G)$. Nodes $u$ and $v$ are arbitrary vertices in $G$. Then the number of even (odd) alternating paths connecting the nodes $\mathrm{u}$ and $\mathrm{v}$ can be specified as ${ }_{p+}(u, v)\left(_{p-}(u, v)\right)$.

Let $G$ be a bigraph with unique 1-factor $M(G)$. Then the labeled bigraph $G^{-1}$ with label $l: E(G) \rightarrow Z$ can be obtained as follows [1, 10-12]:

- $V\left(G^{-1}\right)=V(G)$

- let vertices $u, v \in V\left(G^{-1}\right)$; then the edge $(u, v) \in V\left(G^{-1}\right)$ if and only if ${ }_{p^{+}}(u, v) \neq_{p_{-}}(u, v)$ and $l(u, v)=_{p_{+}}(u, v)-{ }_{p^{-}}(u, v)$.

\section{Examples}

A simple general example to illustrate the described theory has been presented in [5]. A bipartite matrix $B(G)$ is defined to simplify the transcription.

It is obvious that the matrix $B(G)$ is square and it is regular as well. The concrete example can be transformed, according to the rules described above, to the graph structure presented in Fig. 4. The description of vertices corresponds to 1-factor. Since the 1-factor and the alternating paths are very important in the process, it is recommended to mark these components. So, the edges of 1-factor are displayed bold in Figs. 4 and 5. The bipartite matrix of graph $G$ is:

$$
B(G)=\left(\begin{array}{llllllll}
1 & 0 & 0 & 0 & 0 & 0 & 0 & 0 \\
1 & 1 & 0 & 0 & 0 & 0 & 0 & 0 \\
0 & 1 & 1 & 0 & 0 & 0 & 0 & 0 \\
0 & 0 & 1 & 1 & 0 & 0 & 0 & 0 \\
1 & 0 & 0 & 0 & 1 & 0 & 0 & 0 \\
0 & 0 & 0 & 0 & 1 & 1 & 0 & 0 \\
0 & 0 & 0 & 0 & 0 & 1 & 1 & 0 \\
0 & 0 & 0 & 1 & 0 & 0 & 1 & 1
\end{array}\right)
$$

The practical realization of defined rules to follow is very simple. Point No. 1 from Fig. 4 (represents the input to the system) is connected only to the resistor $R_{1}$ (Fig. 3 ) at the beginning part of the scheme (point $1^{\prime}$ in Fig. 4). Thus the value $B_{11}$ is equal to

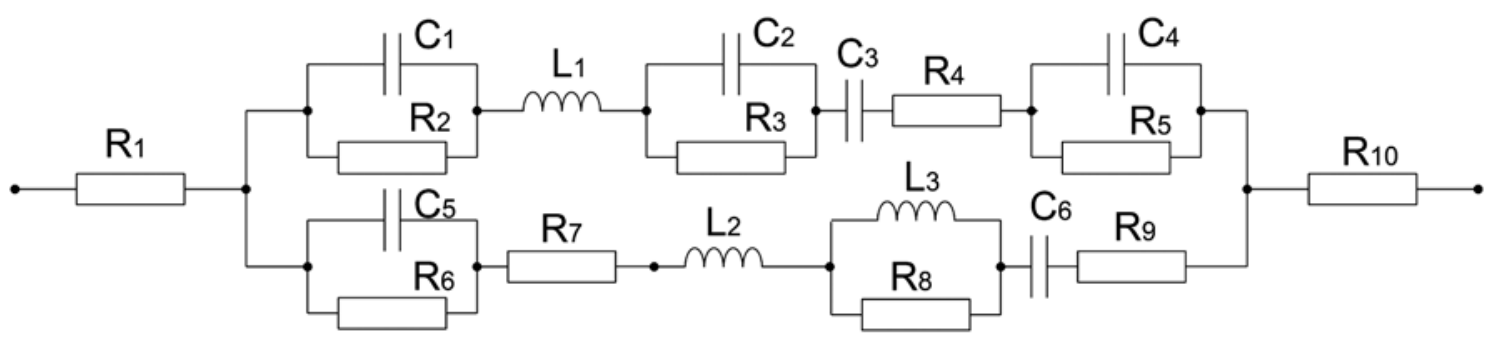

Fig. 3 Example of the electric circuit scheme 
1 , the other values in the first row are equal to 0 . On the other hand, the parallel connection of resistor $R_{2}$ and capacitor $C_{1}$ (represented by point No. 2 in Fig. 4), has an association to points No. $1^{\prime}$ and 2. So the relevant values in $B(G)$ are set to 1 while the others in the second row are set to 0 . This process leads to construction of complete matrix $B(G)$, finished by line No. 8, where $R_{10}$ (point No. 8 in Fig. 4) has an association to points No. 4', 7' and $8^{\prime}$.

When constituting the graph $G^{-1}$ (with bipartite matrix $B^{-1}(G)$ - an inverse matrix to $B(G)$ ) the below described steps have to be followed:

1, the edges of 1-factor are put into the graph $G^{-1}$ and a value of 1 is assigned to them (Fig. 4).

2 , the other edges are included into the graph structure following the algorithm:

- if between two vertices $u^{\prime}$ and $\mathrm{v}$ an alternative path(s) exists, then the number of even ${ }_{p+}\left(u^{\prime}, v\right)$ and odd ${ }_{p-}\left(u^{\prime}, v\right)$ occurrences have to be counted. A new edge will be added in case when ${ }_{p+}\left(u^{\prime}, v\right) \neq{ }_{p-}\left(u^{\prime}, v\right)$ and its value (label) is $l\left(u^{\prime}, v\right)=$ $={ }_{p+}\left(u^{\prime}, v\right)-{ }_{p-}\left(u^{\prime}, v\right)$,

- if not, the edge is not added.

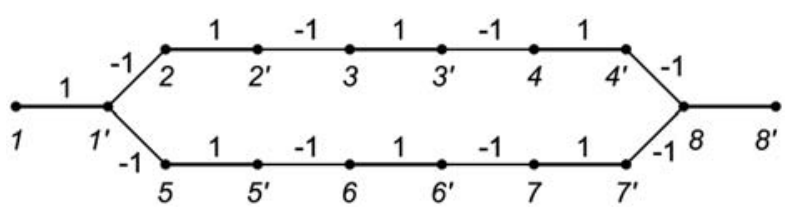

Fig. 4 Structure of graph $G$

The even alternative path has an even number of edges out of 1-factor, while odd has an odd number of those edges. The graph constructed following these rules can be seen in Fig. 5. The corresponding $B\left(G^{-1}\right)$ is:

$$
B\left(G^{-1}\right)=\left(\begin{array}{cccccccc}
1 & 0 & 0 & 0 & 0 & 0 & 0 & 0 \\
-1 & 1 & 0 & 0 & 0 & 0 & 0 & 0 \\
1 & -1 & 1 & 0 & 0 & 0 & 0 & 0 \\
-1 & 1 & -1 & 1 & 0 & 0 & 0 & 0 \\
-1 & 0 & 0 & 0 & 1 & 0 & 0 & 0 \\
1 & 0 & 0 & 0 & -1 & 1 & 0 & 0 \\
-1 & 0 & 0 & 0 & 1 & -1 & 1 & 0 \\
2 & -1 & 1 & -1 & -1 & 1 & -1 & 1
\end{array}\right)
$$

The practical realization of the above described steps is again very easy. For instance, when analyzing the existence of alternative paths to point No. 4, only two of them exist (Fig. 5):

- from $2^{\prime}$ - this includes 3 occurrences, the final value assigned to this path is: 1 , and

- from $1^{\prime}$ - this includes 5 occurrences, the final value assigned to this path is: -1 .

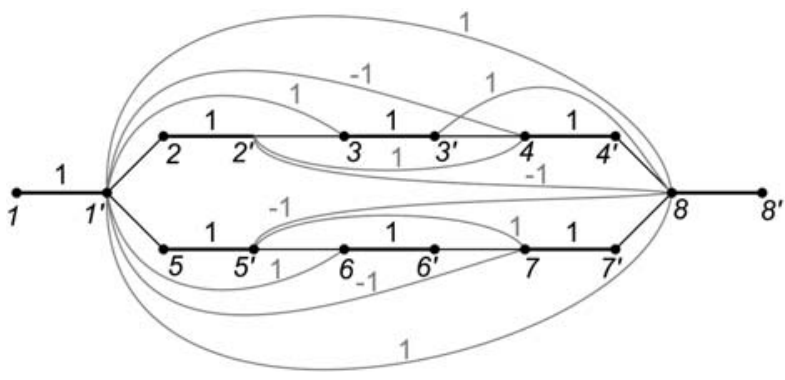

Fig. 5 Paths in $G$

Together with paths:

- from $3^{\prime}$ - assigned as -1 , and

- from $4^{\prime}$ - assigned as 1

the fourth row of $B\left(G^{-1}\right)$ is set to $(-1,1,-1,1,0,0,0,0)$.

The alternative paths illustrated in Fig. 5 represent all available alternatives. The second half of the paths can be easily constructed based on problem symmetry; although this cannot be generalized. Nevertheless, one important remark has to be made in this point: the alternative paths coming to point No. 8 are as illustrated in Fig 5 , but because of the mentioned symmetry case there are two alternative paths from $1^{\prime}$ available. Thus the value of $B_{81}$ is equal to +2 !

Finally, it can easily be proven that the bipartite matrix of graph $G^{-1}$ is an inverse matrix to $B(G)$ :

$$
B^{-1}(G)=B\left(G^{-1}\right)
$$

The procedure for adjacent matrix and its inverse matrix establishment is presented in [5].

At the conclusion of this chapter it has to be written that a fully new principle for obtaining the adjacent matrix has been successfully tested. The bipartite matrix (as a base of the adjacent matrix) is a fundamental component for (almost) any following both numerical and analytical analysis of (but not only) electric circuits. Even though this, it also has to be mentioned that there are certain issues where this technology cannot be applied or where the computational complexity of this kind of solution does not allow its successful implementation.

\section{Conclusion}

This paper is based on the authors' results presented in [5] completed with the latest knowledge and authors' experience. According to these, the base of any numerical method used in technical sciences to analyze and simulate the technical processes is constituted on matrix calculus [1]. This is especially clear for electric circuits used in electrical engineering and power electronics [13-16], where the physical reality and description of the circuit is transformed into a matrix form. The numerical methods in these 
cases are based on inverse matrix calculus. This can be done either in an algebraic or numerical way.

The presented paper discovers a purely new technique to obtain the inverse matrix necessary to construct the sets of equations. This technique is based on graph theory and represents a unique and new approach to numerical solutions of electrical problems. Generally, according to the presented technology, only the number of components is important for building the adjacent matrix. The concrete value of components is not important for this step of the solution and may vary from element to element.

The method itself had been introduced in the paper. The possible way of electric circuit representation by graph structures had been presented, as well as the matrix construction based on the obtained graph. The mathematical background of inverse matrix by graph construction had been presented, including the alternating path influence on matrix system construction.

Compared to the authors' results presented in [5] the background theory was elaborated in a more precise way. The practical example (asynchronous machine) were chosen to demonstrate the theory and possible application of graphs. More complex example is presented here by the authors and a simple solution based on bipartite matrix is offered. This example (Fig. 3) can be considered as an analogue filter used as a basic building block in signal processing. The topology of the filter was chosen to demonstrate the mathematical concept of the designed procedure. To be able to follow the procedures a detailed description of the most important steps is also presented.

The advantages of the presented unique method can be summarized as the following:
- the inverse matrix can be obtained without using either algebraic or numerical technique; the necessity of algebraic description of the task requiring the computation of inverse matrix is completely eliminated,

- graphical (clear) visibility,

- compared to [17] an alternating paths are taken into account,

- a simplified solution based on bipartite matrix is suggested.

The presented method has also several disadvantages. These might sometimes also lead to unsuccessful application of the presented methods:

- necessity of graph scanning and finding the alternating path(s), including the finding the 1 -factor,

- algorithm is valid for bipartite graph structures only.

On the other hand, there is a computational complexity of the method. As has been mentioned above, the method based on alternating paths is a completely new method. The searching of 1 -factor is sufficiently described in the literature, so it is not presented in the paper. The authors describe the principles and offer an example - the question of computational complexity is outside the scope of this paper. Nevertheless, it is clear that the presented method can be successfully used mostly with "sparse" graphs with limited number of edges.

\section{Acknowledgement}

This paper has been supported by the following projects:

Theory of Numbers and Its Application in Fuzzy Modeling, Grant No. Vega 1/0569/12, STU Bratislava, Slovakia.

Developing Skills of Scientific a Research Employees at the CTU in Prague, Grant No. OPPA CZ.2.17/1.1.00/32205, CTU Prague, Czech Republic.

\section{References}

[1] FABO, P., PAVLIKOVA, S.: GSIM - Software for Simulation in Electronics. $14^{\text {th }}$ Intern. Power Electronics and Motion Control Conference, EPE-PEMC 2010, New York: IEEE, 2010, pp. S414-S417, ISBN 978-142447854-5.

[2] ARCHDEACON, D.: Topological Graph Theory - A Survey. [p. 1.]: [Citeseer], 1996, 67 p.

[3] KONAR, R., MICIAN, M.: Numerical Simulation of Residual Stresses and Distortions in Butt Weld in Simulation Programme Sysweld, Communications - Scientific Letters of the University of Zilina, vol. 14, No. 3, pp. 49-54, 2012, ISSN 1335-4205.

[4] DADO, D. et al.: Numerical Investigation of Optical Burst Switching. Communications - Scientific Letters of the University of Zilina, vol. 10, No. 2, pp. 20-24, 2008, ISSN 1335-4205.

[5] PAVLIKOVA, S., MAGA, D., SIMAK, B.: Graph Based Inversed Matrix in Electric Circuits Solutions, Proc. of $9^{\text {th }}$ Intern. Conference, Elektro, 2012. pp. 443-447. ISBN 978-146731179-3

[6] RAFAJDUS, P. et al.: Static and Dynamic Analysis of Linear Switched Reluctance Machine. Communications - Scientific Letters of the University of Zilina, vol. 13, No. 4, pp. 6-11, 2011, ISSN 1335-4205.

[7] PAVLIKOVA, S.: Extending the Class of Matrices Invertible Using Graphs. $6^{\text {th }}$ Conference on Mathematics and Physics at Technical Universities, Brno: UO, 2009, pp. 203-210, ISBN 978-80-7231-667-0.

[8] CVETKOVIC, D., DOOB, M., GUTMAN, I., TORGASEV, A.: Recent Results in the Theory of Graph Spectra. Annals of Discrete Mathematics, Amsterdam : North-Holland, 1988, ISBN 0-444-70361-6.

[9] Godsil, C. D.: Inverses of Trees, Combinatorica, 5, pp. 33-39, 1985.

[10] PAVLIKOVA, S., KRC-JEDINY, J.: On the Inverse and the Dual Index of a Tree. Linear and Multilinear Algebra, vol. 28, pp. 93-109, 1990, ISSN 0308-1087. 
[11] PAVLIKOVA, S.: Application of Eigenvalue Linear Mapping with Utilization of Graph Representation of Linear Mappings. Mechatronika 2009, Trencin : TnUAD, 2009, pp. 99-102, ISBN 978-80-8075-392-4.

[12] PAVLIKOVA, S.: Applications Graphs Spectral Properties in Technical Sciences. Cybernetic Letters, 2011. ISSN 1802-3525

[13] MAGA, D., HARTANSKY, R.: Numerical Solutions. University of Defence, Brno, 2006, 174 p, ISBN 80-7231-130-1.

[14] BAUER, P., VAN DUIJSEN, P.: Challenges and Advances in Simulation. $36^{\text {th }}$ Annual IEEE Power Electronic Specialists. New York: IEEE, pp. 1030-1036, 2005, ISSN: 0275-9306. ISBN 0780390334; 978-078039033-1.

[15] KRUPKA, Z., STEFEK, A.: Method Cooperation in Optimization Process. Mechatronika 2010. Trencin: TnUAD, pp. 21-23, 2010, ISBN 978-808075461-7.

[16] SVOBODA, J., SIMAK, B., ZEMAN, T., HRAD, J.: Educational Activities of the CTU Prague Team in EMC and Support of the EMC Development Problems in the Czech Republic. $13^{\text {th }}$ Intern. Wroclaw Symposium and Exhibition on Electromagnetic Compatibility. Wroclaw : Institute of Telecommunications, pp. 208-211, 1996, ISBN 83-901999-4-7.

[17] FIELDLER, M.: On Inverting Partitioned Matrices. Math. J. 13 (88), pp. 574-586, 1963. 